\section{Les projets de résidence devraient-ils intégrer plus d'un résident?}

\section{LE POUR}

Les normes d'agrément du Conseil canadien de la résidence en pharmacie d'hôpital (CCRPH) exigent que les résidents en pharmacie réalisent un projet en lien avec la pratique pharmaceutique ${ }^{1}$. Les résidents doivent prendre part à l'élaboration du projet et participer à la collecte, à l'analyse et à l'interprétation des données dans le cadre du projet. De plus, ils doivent rédiger un rapport de projet dans un format apte à être publié dans une publication révisée par les pairs, rapport qui sera aussi présenté sous forme d'affiche par les résidents ${ }^{1}$. Les projets de résidence représentent une part importante de la formation des résidents, car ils offrent une occasion unique d'apprendre et de mettre en pratique les bases de la méthodologie de recherche, de démontrer leurs aptitudes en gestion de projet et d'apporter une contribution à la littérature biomédicale. D'ailleurs, l'acquisition de ces compétences de recherche est bien vue par les pharmaciens actifs. En effet, un sondage récent auprès de pharmaciens hospitaliers canadiens exerçant en soins critiques indiquait que $97,2 \%$ des répondants étaient d'avis que leur participation à la recherche est souhaitable et plusieurs $(80,4 \%)$ ont exprimé leur intérêt à y contribuer davantage ${ }^{2}$.

Les normes d'agrément du CCRPH n'indiquent pas si le projet de résidence doit être réalisé par un seul résident ou s'il peut être exécuté par une équipe de résidents. Depuis bon nombre d'années, les résidents du programme de maîtrise en pharmacothérapie avancée de l'Université de Montréal réalisent leur projet de résidence en équipes de deux à cinq personnes, normalement toutes situées dans le même établissement de santé affilié. En fait, depuis plus d'une décennie, tous les projets ont été effectués en équipes. Cette situation tranche nettement avec celle des autres programmes de résidence canadiens qui, normalement, offrent ou même exigent des projets individuels.

Nous reconnaissons les nombreux avantages des projets de résidence menés en équipes, le premier étant la possibilité de développer et d'utiliser une solide méthodologie scientifique, y compris un échantillon de taille adéquate. L'incapacité de produire un échantillon de taille convenable est l'un des inconvénients des projets de résidence réalisés par un seul résident. Augmenter la période de collecte de données en ayant une série de résidents qui recrutent des patients pour le projet rend possible l'obtention d'un échantillon de taille suffisante pour obtenir assez de précision et de puissance statistiques, deux critères essentiels permettant d'inférer des conclusions de qualité. En fait, bon nombre de comités d'examen institutionnels jugent qu'il est contraire à l'éthique de mener une étude dont la puissance statistique est insuffisante. Lorsque différents résidents participent à des projets de recherche pendant plusieurs mois, cela permet aussi de mettre en ouvre des méthodologies de recherche plus complexes, comme celles des études de cohorte prospectives ou des essais cliniques. Enfin, comme ces types de projet ont de meilleures chances d'être publiés, leur potentiel de contribution à l'avancement de la recherche en pharmacie est plus grand. Cela augmente le sentiment de satisfaction non seulement chez les résidents, mais aussi chez les précepteurs et coauteurs de même qu'à l'intérieur des programmes de résidence, des services de pharmacie et des institutions. Une étude récente examinant les taux de publication des projets de résidents canadiens en pratique pharmaceutique entre 1999 et 2009 a montré que le taux global de publication (sous forme de résumé ou de manuscrit) était de $32,2 \%{ }^{3}$. Au cours des trois dernières années de l'étude (2007, 2008 et 2009), le taux de publication sous forme de manuscrit était respectivement de $22,4 \%, 23,2 \%$ et $12,6 \%$. En revanche, à l'Université de Montréal, 46,8\% des projets de résidence en pharmacie ont été présentés sous forme de résumé pendant une période de 11 ans s'échelonnant entre 1997 et $2007^{4}$. Au cours de cette période, $22,6 \%$ des projets ont été publiés, la majorité (82\%) dans des revues indexées ${ }^{4}$. Une recherche dans PubMed des projets de résidence réalisés en 2007, 2008 et 2009 a révélé que respectivement $25 \%, 33 \%$ et $35 \%$ des projets de résidence ont été publiés.

Le deuxième avantage des projets de résidence effectués en équipes est qu'ils reproduisent des situations réelles. La recherche contemporaine exige habituellement une collaboration avec divers professionnels ayant des profils semblables ou différents. Permettre aux résidents en pharmacie de travailler avec des personnes ayant différentes opinions, forces, faiblesses ou différents profils constitue une étape importante du développement de compétences professionnelles. Lors de la réalisation d'un projet collaboratif, l'ensemble des résidents participe à l'élaboration du protocole de recherche, à la collecte et à l'analyse de données, à la présentation des résultats sous forme de résumé, et à la rédaction du rapport sous forme de 
manuscrit destiné à la publication. Par contre, la contribution de chaque résident en ce qui a trait à la collecte et à l'analyse de données peut varier selon le moment de leurs stages de recherche au cours de l'ensemble de la durée du projet ${ }^{5}$. Développer des stratégies permettant d'assurer une collecte rigoureuse et uniforme des données lorsque plusieurs personnes participent à la tâche est un autre avantage précieux d'une formation en recherche, car il est nécessaire d'avoir de bonnes habiletés en communications interpersonnelles. En outre, s'assurer que l'ensemble des membres de l'équipe participe à parts égales peut représenter un défi. Ceci dit, c'est aussi le cas dans les situations réelles de la pratique pharmaceutique et des travaux de recherche. Adapter l'évaluation du projet pour faire en sorte qu'elle comprenne des évaluations individuelles, qui tiennent compte de la participation de chaque résident, ainsi que des évaluations par les pairs (évaluation des résidents par d'autres résidents) permet de surmonter cette contrainte.

Les programmes de résidence en pharmacie peuvent réaliser une utilisation optimale des ressources par l'intermédiaire de projets de résidence en pharmacie effectués en équipes. Le fait de concentrer les ressources en un projet plus grand, plutôt qu'en plusieurs petits projets, peut motiver les précepteurs à y participer. Lorsque les résidents travaillent sur des projets de recherche d'envergure, ils fournissent souvent un apport important au programme de recherche de leur faculté ou de leur directeur clinique ainsi qu'au volet de recherche qui fait partie de la mission du service de pharmacie. Bon nombre de ces projets sont déjà financés par l'intermédiaire du programme de recherche du directeur. Sinon, il est aussi possible d'obtenir le financement approprié du service. Bien que la supervision de projets de résidence en pharmacie puisse exiger beaucoup de temps, les membres de la faculté entrevoient maintenant les bénéfices qu'ils peuvent tirer de cette tâche. Le Programme de formation et de liaison en néphrologie (ProFil) est un exemple récent de ce type de projet; il s'agit d'un travail de recherche qui évalue l'incidence d'un programme de formation et de réseautage en néphrologie dans le but d'améliorer le dépistage et la prise en charge de problèmes liés à la pharmacothérapie par des pharmaciens communautaires. Au total, six équipes de résidents, se succédant sur plusieurs années, ont participé à différents projets au sein du programme, ce qui s'est traduit par de nombreuses publications $s^{6-8}$. Des projets évaluant l'issue et la prise en charge de maladies respiratoires ${ }^{9-11}$ et de la thromboprophylaxie ${ }^{12-14}$ représentent d'autres exemples de réussite.

En conclusion, les projets de résidence en pharmacie comprenant plus d'un résident répondent aux exigences du CCRPH et sont avantageux pour toutes les parties concernées. Les résidents en pharmacie acquièrent une excellente formation en recherche ainsi que d'autres compétences professionnelles, l'établissement (le service de pharmacie de l'hôpital, le centre de recherche institutionnel ou la faculté de pharmacie) est grandement reconnu pour ses programmes de recherche et l'ensemble de la pratique de la pharmacie hospitalière profite des avancées produites par la recherche.

[Traduction par l'éditeur]

References

1. Conseil canadien de la résidence en pharmacie d'hôpital. Normes d'agrément janvier 2010. Ottawa $(\mathrm{ON})$ : Société canadienne des pharmaciens d'hôpitaux; 2009. Publié au www.cshp.ca/programs/residencytraining/FRENCH__2010_CHPRB_Accreditation_Standards.pdf. Consulté le 24 octobre 2013.

2. Perreault MM, Thiboutot Z, Burry LD, Rose L, Kanji S, LeBlanc JM, et coll. Canadian survey of critical care pharmacists' views and involvement in clinical research. Ann Pharmacother. 2012;46(9):1167-73.

3. Hung M, Duffett M. Canadian pharmacy practice residents' projects: publication rates and study characteristics. Can J Hosp Pharm. 2013;66(2):86-95.

4. Perreault MM, Turgeon N, Williamson DR, Lalonde L. Ten years of residency projects: a review [résumé]. Am J Pharm Educ. 2008;72:29.

5. Egan G, Seaton A. Completing a residency project involving 2 residents [lettre]. Can J Hosp Pharm. 2012;65(4):323-4.

6. Legris MÈ, Séguin NC, Desforges K, Sauvé P, Lord A, Bell R, et coll. Pharmacist Web-based training program on medication use in chronic kidney disease patients: impact on knowledge, skills, and satisfaction. $J$ Contin Educ Health Prof. 2011;31(3):140-50.

7. Desrochers JF, Lemieux JP, Morin-Bélanger C, Paradis FS, Lord A, Bell R, et coll. Development and validation of the PAIR (Pharmacotherapy Assessment in Chronic Renal Disease) criteria to assess medication safety and use issues in patients with CKD. Am J Kidney Dis. 2011;58(4):527-35.

8. Guay M, Ricard S, Letendre S, Clément V, Lord A, Bell R, et coll. Connaissances et compétences des pharmaciens communautaires et qualité de la pharmacothérapie chez les patients atteints d'insuffisance rénale chronique. Pharmactuel. Sous presse.

9. Thuot M, Coursol MA, Nguyen S, Lacasse-Guay V, Beauchesne MF, Fillion A, et coll. Impact of obesity on perinatal outcomes among asthmatic women. Can Respir J. 2013;20(5):345-50.

10. Beauchesne MF, Bercier D, Julien-Baker F, Lalonde L, Boileau R, Blais L. Community pharmacy-based medication assessment program for asthma and chronic obstructive pulmonary disease. Can Pharm J. 2012;145(2):70-1.

11. René-Henri N, Khamla Y, Nadaira N, Ouellet C, Blais L, Lalonde L, et coll. Community pharmacists' interventions in asthma care: a descriptive study. Ann Pharmacother. 2009;43(1):104-11.

12. Vincent PD, Albert M, Champagne MC, Zikos T, Boulanger I, Blais L, et coll. Factors influencing enoxaparin anti-Xa activity in surgical critically ill patients. J Crit Care. 2011;26(4):347-51.

13. Bernier MC, Desjardins K, Filiatrault J, Sauriol MA, Martineau J, Gilbert E, et coll. Implementation and evaluation of a pharmacy-led thromboprophylaxis campaign in a community hospital. J Thromb Thrombolysis. 2011;32(4): 431-8.

14. Bazinet A, Almanric K, Brunet C, Turcotte I, Martineau J, Caron S, et coll. Dosage of enoxaparin among obese and renal impairment patients. Thromb Res. 2005;116(1):41-50.

David R. Williamson, B. Pharm., M.Sc., BCPS

Professeur agrégé de clinique

Lyne Lalonde, B. Pharm., Ph. D.

Professeure titulaire

Faculté de pharmacie

Université de Montréal

Montréal, Québec

David Williamson est aussi un pharmacien exerçant en soins critiques au sein du service de pharmacie de I'Hôpital du Sacré-Cœur de Montréal, à Montréal, au Québec. Lyne Lalonde est aussi chercheuse au Centre de recherche du Centre hospitalier de I'Université de Montréal, à Montréal, au Québec.

Intérêts concurrents : Lyne Lalonde a obtenu des subventions provenant des Instituts de recherche en santé du Canada, d'Amgen et de LEO Pharma pour des projets apparaissant dans cet article. Aucun intérêt concurrent déclaré par M. Williamson. 


\section{LE CONTRE}

Le programme de résidence offert aux pharmaciens qui souhaitent exercer en établissement de santé a plusieurs visées; en plus des objectifs cliniques, il vise à développer et à exécuter des projets de recherche qui portent sur un aspect scientifique, clinique ou administratif de la pratique de la pharmacie en établissement de santét, ${ }^{1,}$.

À la Faculté de pharmacie de l'Université Laval, la grande majorité des projets de résidence (Maîtrise en pharmacothérapie avancée) sont réalisés par un seul résident. Pourquoi? Nos partenaires de projets sont les pharmaciens issus des établissements de santé qui offrent le volet clinique du programme de résidence. Comme le mentionne Barletta ${ }^{3}$, il est crucial au départ que le projet proposé soit le "bon projet » de recherche. Ainsi, le type de projet et son envergure doivent répondre aux besoins de nos milieux. Notre expérience démontre que les recherches portant sur l'évaluation d'une pratique, la concordance avec un protocole ou une ligne directrice, le développement d'outils, l'évaluation de l'utilisation d'un médicament, dont la gestion d'un effet indésirable, ou une étude pharmacocinétique ainsi que la réponse à des questions cliniques sont des exemples de projets qui non seulement répondent au besoin de nos partenaires mais sont réalisables par un seul résident ${ }^{4-9}$.

Selon Barletta ${ }^{3}$, les projets qui ne peuvent se réaliser au complet durant l'année de résidence ne sont pas souhaitables. Bien que les essais cliniques à répartition aléatoire et prospectifs constituent la méthodologie la plus rigoureuse pour répondre à une question de recherche, ils nécessitent plusieurs années et ne sont donc pas des choix réalistes de par la contrainte de temps ${ }^{3}$. En outre, la réalisation d'un projet de recherche ne doit pas se faire au détriment de l'acquisition de compétences cliniques. En effet, le programme est fort chargé et le temps consacré au projet est limité 2 . Barletta soutient l'idée de mettre sur pied des projets qui ne devraient pas se poursuivre sur plus d'une année pour que le résident puisse être exposé à toutes les étapes du projet de recherche ${ }^{3}$. Celui qui réalise seul son projet acquiert donc des connaissances et des compétences spécifiques à toutes les étapes de la réalisation (définition du sujet, formulation des objectifs opérationnels, planification de ses activités selon son horaire, obtention des autorisations nécessaires, développement des outils de collecte, collecte et analyse des données, interprétation des résultats et présentation orale et écrite). Le résident sera ainsi apte à développer une réflexion critique et logique lors de la planification de futurs projets, à démontrer sa capacité à gérer un projet ainsi qu'à partager des connaissances.

Pour les superviseurs, la réalisation du projet mené par un seul résident comporte des avantages certains. La répartition de la charge de travail associée au projet s'en trouve simplifiée. Ainsi, la question de la contribution relative de chaque résident au projet ne se pose pas. Un autre avantage découle du fait que la collecte de données ne risque pas de subir des variations dues au nombre d'évaluateurs. Ces problèmes imposent des limites aux résultats d'un projet réalisé par plusieurs chercheurs ${ }^{10}$.
Par ailleurs, la réalisation d'un projet par un seul résident peut prévenir certains problèmes de communication. Egan et Seaton rapportent d'ailleurs que la communication peut être problématique lorsque les opinions, le style de communication ainsi que les visions different entre les résidents ${ }^{10}$. De plus, les projets menés par un seul résident permettent d'éviter les conflits de personnalité entre des résidents ayant des profils peu compatibles ou une façon différente d'envisager la collaboration ${ }^{10}$. Il faut également mentionner le danger d'un désistement d'un résident, ce qui pourrait remettre en question la viabilité ou la faisabilité du projet entrepris à deux ou à plusieurs. La diffusion des résultats du projet de recherche représente une partie essentielle de la formation des résidents ${ }^{1,11}$. Hung et Duffett ${ }^{11}$ ont décrit le type de projets réalisés au sein des programmes de résidence au Canada (Québec non inclus) et les taux de publication par résumé et par article. Selon leur rapport, les projets publiés ainsi que les thèmes de recherche sont très variés et généralement réalisables par un seul résident ${ }^{11}$. De plus, la majorité des projets publiés $(89,1 \%)$ ont chacun été réalisés dans un seul centre. Le tableau 1 présente quelques exemples de projets pilotés par un seul résident au sein de notre faculté et ayant mené à une publication au cours des dernières années ${ }^{4-9}$.

La réalisation d'un projet par une seule personne ne cause en outre aucune ambiguïté sur le choix du premier auteur de la publication. Une telle reconnaissance n'est pas à négliger, puisqu'elle procure certainement un avantage au résident qui décide de poursuivre une carrière en recherche.

Les exemples de projets cités au tableau 1 et réalisés par un seul résident permettent l'atteinte des exigences du Conseil canadien de la résidence en pharmacie d'hôpital $(\mathrm{CCRPH})^{1}$ en ce qui a trait au projet de résidence, dont :

- Le résident doit prendre part l'élaboration d'un projet et participer à la récolte, à l'analyse et à l'interprétation des données dans le cadre du projet.

- Le résident doit préparer un rapport écrit du projet dans un format apte à être publié dans une publication approuvée par des collègues.

- Le résident doit présenter et défendre les résultats de son projet.

La présence d'un seul résident dans certains établissements isolés limite aussi la réalisation collective d'un projet de recherche. Deux de nos établissements se situent physiquement à plus de $220 \mathrm{~km} \mathrm{du}$ centre urbain où se trouvent nos hôpitaux universitaires. Cette réalité géographique complique la réalisation d'un projet de recherche en équipe.

De plus, les établissements de santé qui accueillent nos résidents possèdent des expertises cliniques et des populations de patients très différentes. À titre d'exemple, l'Institut universitaire de cardiologie et de pneumologie de Québec est un centre surspécialisé qui offre des soins tertiaires en cardiologie et en pneumologie $^{12}$. La réalité de l'Hôpital régional du Centre de santé et de services sociaux de Rimouski-Neigette est tout autre, il assure notamment à la population des services de deuxième 


\section{Tableau 1. Exemples de projets publiés et réalisés par un seul résident (en collaboration avec des non-résidents)}

\begin{tabular}{|c|c|c|}
\hline Type & Auteurs & Titre \\
\hline Gestion d'un effet indésirable & Tessier et coll. ${ }^{4}$ & $\begin{array}{l}\text { "Minocycline en prévention des éruptions cutanées à } \\
\text { l'erlotinib pour les patients atteints d'un cancer pulmonaire } \\
\text { non à petites cellules» }\end{array}$ \\
\hline Évaluation d'un protocole & $\begin{array}{l}\text { Pellerin et coll. }{ }^{5} \\
\text { de Maupeou } \\
\text { d'Ableiges et coll. }^{6}\end{array}$ & $\begin{array}{l}\text { "Évaluation du nomogramme d'ajustement de l'héparine non } \\
\text { fractionnée intraveineuse de l'Hôpital Laval » } \\
\text { "Utilisation transitoire de la daltéparine lors de l'interruption de } \\
\text { l'administration de warfarine en vue d'une chirurgie cardiaque } \\
\text { évaluation d'une nouvelle procédure de désanticoagulation » }\end{array}$ \\
\hline Étude pharmacocinétique & Simoneau et coll. ${ }^{8}$ & $\begin{array}{l}\text { "Circadian variations in the pharmacokinetics of a new } \\
\text { microemulsion formulation of cyclosporine in cardiac } \\
\text { transplant recipients* » } \\
\text { "Effect of prophylactic dalteparin on anti-factor Xa levels in } \\
\text { morbidly obese patients after bariatric surgery† » }\end{array}$ \\
\hline Réponse à une question clinique & Lessard et coll. ${ }^{9}$ & $\begin{array}{l}\text { "Votre expérience avec l'estimation de la fonction rénale selon } \\
\text { différentes formules mathématiques » }\end{array}$ \\
\hline
\end{tabular}

ligne $^{13}$. Notre distance géographique avec l'établissement ou sa mission spécialisée favorisent les projets de recherche menés par un seul résident.

En conclusion, la réalisation d'un projet de résidence par un seul résident répond aux exigences du CCRPH et aux besoins de nos partenaires en clinique en plus de permettre au résident d'être exposé à toutes les étapes du projet de recherche. Ainsi, après avoir réalisé leur projet, les résidents en pharmacie pourront aborder les problématiques d'un projet de recherche avec méthode et rigueur. Les types de projets réalisés et les thèmes de recherche sont diversifiés, et la diffusion des résultats, notamment par la publication sous forme d'articles, est vivement encouragée. La présence d'un seul résident dans certains établissements, les missions différentes des établissements et la réalité géographique justifient la réalisation de projet par un seul résident.

\section{References}

1. Conseil canadien de la résidence en pharmacie d'hôpital. Normes d'agrément, janvier 2010. Ottawa (ON) : Société canadienne des pharmaciens d'hôpitaux; 2009. Publié au www.cshp.ca/programs/residencytraining/ FRENCH_-_2010_CHPRB_Accreditation_Standards.pdf. Consulté le 16 septembre 2013.

2. Maîtrise en pharmacothérapie avancée : description du programme de deuxième cycle. Québec (QC) : Université Laval, Faculté de pharmacie; 2013. Publié au https://www.pha.ulaval.ca/cms/site/pha/pharmacie/programmespharmacie/cycles-superieurs/maitrise-pharmacotherapie-avancee. Consulté le 30 octobre 2013.

3. Barletta JF. Conducting a successful residency research project. Am J Pharm Educ. 2008;72(4):article 92 (sept pages).

4. Tessier JF, Gagnon PY, Côté J, Drolet B. Minocycline en prévention des éruptions cutanées à l'erlotinib pour les patients atteints d'un cancer pulmonaire non à petites cellules. Pharmactuel. 2013;46(2):78-86.

5. Pellerin J, Taillon I, Bélanger PM, Méthot J. Évaluation du nomogramme d'ajustement de l'héparine non fractionnée intraveineuse de l'Hôpital Laval. Pharmactuel. 2008;41(5):277-83.

6. de Maupeou d'Ableiges D, Daleau P, Voisine P, Châteauvert N. Utilisation transitoire de la daltéparine lors de l'interruption de l'administration de warfarine en vue d'une chirurgie cardiaque : évaluation d'une nouvelle procédure de désanticoagulation. Pharmactuel. 2008;41(4):208-17.

7. Châteauvert N, Côté $H$. Circadian variations in the pharmacokinetics of a new microemulsion formulation of cyclosporine in cardiac transplant recipients. Pharmacotherapy. 1998;18(2):364-70.

8. Simoneau MD, Vachon A, Picard F. Effect of prophylactic dalteparin on anti-factor Xa levels in morbidly obese patients after bariatric surgery. Obes Surg. 2010;20(4):487-91.

9. Lessard S, Blanchet M, Morin N, Lefebvre J. Votre expérience avec l'estimation de la fonction rénale selon différentes formules mathématiques. Pharmactuel. 2012;45(1):43-51.

10. Egan G, Seaton A. Completing a residency project involving 2 residents [lettre]. Can J Hosp Pharm. 2012;65(4):323-4.

11. Hung M, Duffett M. Canadian pharmacy practice residents' projects: publication rates and study characteristics. Can J Hosp Pharm. 2013;66(2):86-95.

12. À propos de l'Institut : mission. Québec (QC) : Institut universitaire de cardiologie et de pneumologie de Québec; 2010. Publié au www.iucpq.qc.ca/apropos-mission.asp. Consulté le 30 octobre 2013.

13. Notre organisation : la mission. Rimouski (QC) : Centre de santé et de services sociaux de Rimouski-Neigette; (C) 2003-2013. Publié au www.chrr.qc.ca/organisation/mission/index.php. Consulté le 30 octobre 2013.

Julie Méthot, B. Pharm., Ph. D.

Professeure adjointe

Directrice du programme de maîtrise en pharmacothérapie avancée

Chantale Simard, B. Pharm., Ph. D.

Professeure titulaire

Faculté de pharmacie

Université Laval

Québec, Québec

Julie Méthot est également pharmacienne clinicienne à l'Institut universitaire de cardiologie et de pneumologie de Québec, Québec, Québec. Chantale Simard est également chercheuse au Centre de recherche, Institut universitaire de cardiologie et de pneumologie de Québec, Québec, Québec.

Intérêts concurrents : Julie Méthot est rédactrice en chef de la revue Pharmactuel. Aucun intérêt concurrent déclaré par Chantale Simard. 